\title{
Hérédité et atavisme criminel dans les romans d'Hector Malot
}

\section{Ivan Kiriow}

\section{(2) OpenEdition \\ 1 Journals}

Édition électronique

URL : http://journals.openedition.org/aes/942

DOI : 10.4000/aes.942

ISSN : 2258-093X

Éditeur

Laboratoire LISAA

Référence électronique

Ivan Kiriow, "Hérédité et atavisme criminel dans les romans d'Hector Malot », Arts et Savoirs [En ligne], 7 | 2016, mis en ligne le 12 décembre 2016, consulté le 20 avril 2019. URL : http:// journals.openedition.org/aes/942; DOI : 10.4000/aes.942

Ce document a été généré automatiquement le 20 avril 2019.

Centre de recherche LISAA (Littératures SAvoirs et Arts) 


\title{
Hérédité et atavisme criminel dans les romans d'Hector Malot
}

\author{
Ivan Kiriow
}

1 La postérité n'a guère été juste avec Hector Malot (1830-1907). On ne se souvient souvent de lui que comme l'auteur de Sans Famille (1878), et comme un feuilletoniste sans envergure. En dehors d'une poignée de connaisseurs, les quelque soixante romans qu'il a signés ${ }^{1}$ sont à peu près oubliés. Il a pourtant abordé une extraordinaire variété de thèmes et de milieux, avec un art consommé de romancier. Il fut une voix importante de son époque et son point de vue sur de nombreuses questions mérite un examen plus approfondi ${ }^{2}$. Écrivain réaliste, proche du courant naturaliste mais foncièrement indépendant, Malot puisait volontiers dans les sciences de son époque. Son approche dans ce domaine était certes moins ostentatoire et systématique que celle de Zola, mais il partageait avec ce dernier de nombreuses préoccupations relatives aux sciences du vivant. Les deux romanciers ont d'ailleurs plusieurs fois été rapprochés et comparés sur ce point, et ont été souvent la cible des mêmes critiques ${ }^{3}$.

2 Je m'intéresserai dans cet article au traitement des théories de l'hérédité dans l'œuvre de Malot, et en particulier à sa représentation de la thèse du criminel atavique. Défendue par le médecin italien Cesare Lombroso, cette théorie, qui considérait certains criminels - les "criminels-nés » - comme des résurgences du passé évolutif de l'humanité, avait notamment été mise en scène dans le roman de Zola La Bête humaine (1890).

3 Nous verrons comment Malot, loin de se borner à utiliser fidèlement les thèses de l'anthropologie criminelle, a proposé sa propre version des rapports entre meurtre et atavisme. Certes, sa création s'est nourrie aux mêmes sources où ont puisé Lombroso et ses confrères : les découvertes sur la préhistoire, une certaine interprétation la théorie de l'évolution et des données ethnologiques. Mais elle fut aussi modelée par la philosophie et la vision du monde personnelles de l'auteur. Et loin de suivre ou de copier Zola sur ce terrain, Malot ferait au contraire figure de précurseur de La Bête humaine, ou plutôt de catalyseur, dans un débat où se firent entendre plusieurs voix remarquables. 


\section{L'hérédité chez Malot : le refus du fatalisme}

4 Comme Zola - bien que dans une moindre mesure - Malot a traité à maintes reprises la question de l'hérédité dans ses romans. La critique de la médecine aliéniste et de l'institution asilaire ayant été l'un de ses chevaux de bataille, il a évidemment eu à aborder la question de l'hérédité des maladies mentales, qui était comme la matrice et le paradigme de toutes les théories " anté-mendeliennes " ${ }^{4}$ de l'hérédité. Malot a en effet été salué par plusieurs historiens comme un fer de lance de la littérature antialiéniste. Il a consacré trois de ses romans à la question de la folie et - ce qui le préoccupait plus encore - au problème posé par la législation sur l'internement à la demande d'un tiers, dont les modalités étaient prescrites par la loi sur les asiles, dite « loi de 1838 ».

Dans le triptyque antialiéniste de Malot, Le mari de Charlotte (1875) ${ }^{5}$, encadré chronologiquement par Un beau-frère (1869) et Mère (1890), apparaît comme la pièce mineure. Elle est d'ailleurs expédiée par Aude Fauvel qui, dans l'article qu'elle a consacré à l'aliénisme et au monde des asiles dans l'œuvre de Malot $^{6}$, n'y voit qu'une redite d'Un beau-frère ${ }^{7}$. Il est vrai que la question de l'internement abusif y est moins centrale que dans les deux autres romans, mais elle est aussi traitée avec plus d'ambiguïté. Et l'élément nouveau qui instaure cette incertitude est celui qui nous intéresse ici tout particulièrement : l'hérédité.

6 Hérédité et aliénisme étaient intimement liés au $\mathrm{XIX}^{\mathrm{e}}$ siècle : la transmission à la descendance de diverses maladies, avec une prédilection marquée pour les affections mentales, était le modèle de toutes les tentatives pour théoriser l'hérédité, menées presque exclusivement par des médecins aliénistes, Prosper Lucas en tête ${ }^{8}$. Et la peur de la folie était souvent liée à celle de la folie héréditaire, dans sa propre famille comme dans celles avec lesquelles on était susceptible d'être lié par le mariage : cette double hantise traverse notamment tout le cycle des Rougon-Macquart.

7 Or la question de l'hérédité était la grande absente du réquisitoire anti-aliéniste d'Un beau-frère. ${ }^{9}$. Dans Le mari de Charlotte au contraire, elle est indissociable de celle de l'aliénation. Hanté par la folie de sa mère, qui l'a menée au suicide, Emmanuel Narbanton tait ce lourd passif héréditaire à son épouse, Charlotte, qui l'a épousé pour satisfaire le désir de son père mourant, alors qu'elle aurait voulu s'unir à son cousin Georges Saffarel. Savant amateur passionné et désintéressé, $M$. de la Héraudière, le père de Charlotte, qui a pourtant recueilli et élevé George comme son fils, craint que le jeune homme, un avocat ambitieux et égoïste, ne rende sa fille malheureuse.

8 Mais Saffarel, qui était pourtant l'ami de Narbanton et qui avait présenté ce dernier à La Héraudière et à sa fille, n'a pas oublié la jeune femme, ni la façon dont il a été éconduit. Quand le couple revient en France après une très longue lune de miel, il révèle à Charlotte, qui s'inquiète du comportement de son époux, le terrible secret de son ascendance. Il se lance alors dans une docte plaidoirie sur les dangers de l'hérédité des maladies mentales ${ }^{10}$.

9 Certains critiques ont reproché à Malot l'invraisemblance du tableau clinique de la folie de Narbanton, qui ne correspondrait à aucune pathologie répertoriée ${ }^{11}$. Mais les incohérences flagrantes dans les descriptions de la maladie mentale sont surtout liées à l'ambiguïté qui entoure la folie du jeune homme. À la lecture du roman il est pratiquement impossible de trancher : est-il réellement malade ? Seulement perturbé par 
le souvenir de sa mère, ainsi que par sa jalousie envers Georges? Est-il un simulateur, comme il le suggère lui-même à la fin du roman ${ }^{12}$ ?

Quoi qu'il en soit, l'indécidabilité de cette aliénation entraîne celle de l'hérédité de cette folie. Le plus souvent, la question générale de l'hérédité est d'ailleurs traitée dans l'œuvre de Malot avec la même circonspection. Par sa place centrale dans le triptyque antialiéniste, Le Mari de Charlotte est donc un pivot: écartée d'Un Beau-Frère, l'hérédité est devenue évidente dans Mère, où le véritable fou est stigmatisé comme "dégénéré » même si la fatalité de l'hérédité des maladies mentales est nuancée par le personnage de l'écrivain Darlot ${ }^{13}$, double romanesque de l'auteur ${ }^{14}$.

\section{L'atavisme du crime dans l'œuvre de Malot : la menace ancestrale}

11 Il y a bien lieu de parler des théories, et non de la théorie de l'hérédité, au XIX siècle. Car jusqu'à la redécouverte des lois de Mendel au début $d u x^{e}$, on est plutôt confronté, malgré les déclarations de principe, à une accumulation d'études de cas qu'à une doctrine unifiée. Dans ce foisonnant paysage, nous allons nous intéresser particulièrement à un thème qui cristallise toutes les peurs et les questions liées à l'hérédité : l'atavisme du penchant au meurtre.

Théorisée par le médecin aliéniste et criminaliste italien Cesare Lombroso dans son livre $L^{\prime}$ Homme criminel (L'Uomo criminale, traduit en français en 1887) ${ }^{15}$, la figure du criminel atavique, résurgence du passé préhistorique de l'homme, a trouvé une incarnation romanesque saisissante dans le Jacques Lantier de La Bête humaine (1890). Pourtant le binôme Lombroso/Zola, souvent étudié16, ne résume pas à lui seul l'histoire d'une doctrine scientifique élevée au rang de mythe littéraire et populaire. Deux ans avant que Zola fasse paraître son grand roman de crime, son confrère Malot publia quant à lui un diptyque sur le thème du meurtre et de ses conséquences: Conscience (1888) et Justice (1889). La réaction de la critique - littéraire mais aussi philosophique - au premier de ces romans fut l'occasion d'une polémique sur la théorie du "criminel-né » (ainsi que Lombroso désigne le criminel par atavisme), qui va influencer en retour le romancier dans l'écriture du second volet. Cette controverse " prépare » et annonce à plus d'un titre la réception du roman de Zola et de son meurtrier surgi du fond des âges.

\section{Le médecin et le crime : genèse d'une mini-série romanesque}

Plusieurs romans de Malot forment des séries, souvent signalées par un sous-titre commun. Dans le cas de Conscience et Justice, ces deux œuvres constituent de toute évidence un diptyque, le second roman étant la suite du premier. Mais on peut aussi les relier à un troisième livre, paru dix ans avant et dont le rapport avec les deux autres n'est que thématique et antithétique. Ces trois livres présentent en effet des personnages de médecins brillants, confrontés au crime et à ses conséquences juridiques et morales. En 1879 - un an après Sans Famille - Malot avait signé un roman intitulé Le docteur Claude, histoire d'un «bon» docteur, Étienne Claude, luttant contre l'adversité. Après avoir quitté Paris et ses rêves de gloire scientifique, il s'est installé dans une petite ville, où il est en butte à toutes les mesquineries, à l'ignorance et aux préjugés. Alors qu'il 
commençait à être accepté, il est accusé à tort du meurtre de sa femme. En réalité, celle-ci a été empoisonnée par Nathalie, sa propre cousine, une ancienne maîtresse jalouse de Claude. Après un procès éprouvant à l'issue duquel il est condamné à la peine capitale, le docteur est finalement sauvé par le suicide et l'aveu posthume de la véritable coupable. $\mathrm{Si}$ Conscience et Justice forment un ensemble indépendant, ce roman en deux épisodes est aussi comme le négatif du Docteur Claude. Les héros de ces deux histoires, les docteurs Étienne Claude et Victor Saniel, sont à la fois proches et opposés. Ils sont tous deux décrits comme des médecins talentueux, praticiens habiles et probes, mais aussi comme des chercheurs brillants.

14 Certes, Malot ne précise nulle part la nature exacte des recherches du docteur Claude. Tout au plus sait-on qu'il est passé maître dans le maniement d'un instrument qui était encore, étonnamment, peu connu des médecins français: le microscope. Ses connaissances dans ce domaine lui permettent même de sauver deux innocents de la peine capitale par sa contre-expertise dans un procès pour meurtre, ce qui lui vaudra autant de rancunes que d'admiration.

En revanche, le romancier est moins avare de détails sur l'orientation des recherches de Saniel. L'Auvergnat ambitieux " monté » à Paris est un pionnier de la microbiologie ${ }^{17}$, un champ d'investigation scientifique ouvert par les triomphes de Pasteur. Et lorsqu'il gratifie sa maitresse Philis d'un exposé très succinct de son domaine de recherches, il oppose clairement la théorie microbienne à l'hérédité : si le triomphe de la première ne condamne pas sans appel la seconde, elle sonne le glas de son hégémonie dans le domaine de la pathologie ${ }^{18}$.

16 En tant que médecin, Saniel ne fait qu'effleurer la question du rôle de l'hérédité, elle apparait comme secondaire, même si les cas où elle s'applique ne sont pas anodins : dans Conscience, c'est à sa propre personne (sa tendance au somnambulisme) ${ }^{19}$; dans Justice, il s'agit pour lui de déterminer si deux frères, qui sont d'abord de simples patients mais deviendront par la suite ses beaux-fils, peuvent être atteints par la phtisie qui a emporté leur père ${ }^{20}$. L'hérédité est toujours problématique, jamais systématique, elle n'est qu'une possibilité médicale parmi d'autres.

Dans Le docteur Claude, l'hérédité est d'abord présentée comme un fait allant de soi, une explication presque évidente de tous les traits de caractère possibles, invoquée aussi bien par Claude s'adressant à son ancien camarade Lajardie $^{21}$ que par ce dernier ${ }^{22}$. Mais le médecin est par ailleurs confronté à un cas supposé d'hérédité pathologique qui s'avère vite pour lui une question de vie ou de mort, littéralement et à plusieurs titres. Nathalie, tente de le persuader que sa cousine Véronique est condamnée par une cardiopathie héréditaire, afin de l'éloigner de celle qu'elle pressent à juste titre comme une possible rivale. Mais il épouse tout de même Véronique, que Nathalie empoisonne dans l'espoir de le reconquérir. La possibilité qu'il ait eu connaissance d'une maladie héréditaire devient une circonstance aggravante, suggérant qu'il aurait fait un mariage d'intérêt en espérant hériter rapidement de la fortune de son épouse.

\section{Les germes de l'atavisme, d'Un beau-frère au Docteur Claude et à Justice}

Comme c'est le cas pour Zola, Malot n'a pas attendu Lombroso pour évoquer le spectre de l'atavisme criminel, qui puise largement dans le darwinisme social, les découvertes sur les 
origines préhistoriques de l'homme, et doit aussi beaucoup aux interrogations sur le fond obscur de l'âme humaine, qui se cristalliseront notamment dans le "retour du refoulé » freudien.

Dans Un Beau-Frère déjà, la figure de l'aliéné criminel était associée à l'animalité. Cénéri, le héros malheureux du roman, interné à tort dans un asile d'aliénés, y fait la rencontre effrayante d'un meurtrier compulsif, dont il avait « déjà remarqué » la « face ignoble » et les « yeux louches ${ }^{23}$ :

Est-il possible qu'on mette de pareils monstres en contact avec des hommes comme le capitaine Bourdon ou M. d'Auvers ? [...] Faut-il les tuer ? Non, assurément ; mais puisque ce sont des bêtes féroces, et rien que cela, les traiter comme des bêtes féroces du Jardin des Plantes. Cela vous donnerait trop de peine et vous coûterait trop cher? J'entends. Ne dites pas alors que les fous sont pour vous des malades. À des malades on n'impose pas la compagnie des brutes. ${ }^{24}$

Dans Le mari de Charlotte, le personnage de La Héraudière est un savant amateur mais passionné, qui consacre son temps libre de retraité à deux chantiers scientifiques: l'acclimatation d'espèces végétales exotiques dans le jardin de sa propriété bretonne, et les fouilles archéologiques dans un site proche, un «kjoekken-moedding» («débris de cuisine » en danois, ainsi que le paléontologue amateur l'explique à son neveu et fils adoptif George Saffare ${ }^{25}$ ). Bien qu'elles restent en marge de l'intrigue principale du roman, la question des origines de l'homme et la querelle du transformisme sont souvent évoquées. Pour illustrer l'intransigeance de son oncle dans les questions scientifiques, Saffarel raconte à Charlotte une brouille entre le savant amateur et un de ses plus proches amis :

S'il s'est fâché avec M. Falcot, qui pendant vingt années a été son ami intime, le compagnon de ses travaux, c'est parce que celui-ci a refusé d'admettre que les hommes descendaient des singes. [...] « Celui qui ne reconnaît pas la pluralité des races humaines associée à l'idée du développement progressif des êtres ne peut pas être mon ami, » me dit-il en refermant la porte sur le dos de M. Falcot, qui venait de sortir exaspéré. Et depuis, malgré les tentatives faites par des tiers, mon oncle n'a jamais voulu revoir son ancien ami. ${ }^{26}$

Alors qu'il présente son ami Emmanuel Narbanton - qui n'est pas encore son rival auprès de Charlotte - à son oncle, il le met ainsi en garde :

Quand il se lancera dans des explications sur l'homme primitif et sur ses découvertes, tâche de penser à autre chose.

- Mais pas du tout, l'homme primitif n'a rien qui m'effraye.

- Alors, tu vas faire la conquête de mon oncle [...]. ${ }^{27}$

Enfin, lorsqu'il entend Martin, le domestique de M. de la Héraudière, chanter des cantiques en l'absence de son maître, l'avocat ironise in petto :

- Voilà Martin qui proteste contre la théorie de la transformation de l'espèce, dit-il

en riant, et contre l'homme-singe. ${ }^{28}$

Ces allusions restent anecdotiques et Malot ne dialectise pas le débat sur les origines simiennes de l'homme. De nombreux indices laissent cependant penser qu'il s'était beaucoup documenté sur ces questions : il cite plusieurs noms d'auteurs ayant écrit sur le sujet $^{29}$, et les mésaventures de $\mathrm{M}$. de la Héraudière (il est accusé par un journal local d'avoir été le jouet d'un canular) rappellent celles de Boucher de Perthes lors de sa découverte de «l'homme fossile». Quant à la question des rapports entre crime et folie, en particulier dans ses aspects juridiques, elle n'est évoquée qu'en passant par Narbanton lui-même, dans un passage déjà cité de la fin du roman, où il jette le doute sur la réalité de l'aliénation mentale pour laquelle il a pourtant été interné30. 
23 Dans La Fille de la Comédienne (1875), l'atavisme ne prend pas le visage dramatique et effrayant du criminel-né, il concerne une particularité sensorielle plutôt triviale, mais il est présenté comme un fait d'hérédité avéré31, avec une certitude qui vaudra à Malot les quolibets de la critique antinaturaliste ${ }^{32}$.

24 Dans Le docteur Claude, qui, nous l'avons dit, forme avec le diptyque Conscience/Justice les deux faces d'un même ensemble romanesque sur les médecins et le crime, l'idée d'atavisme criminel est évoquée à propos de Nathalie, l'empoisonneuse qui tue Véronique, sa cousine et la femme de son ancien amant claude :

Son premier mouvement l'avait poussée à étrangler de ses mains sa rivale, mais cela avait été purement instinctif; c'était un acte bestial; de notre temps, où l'on ne se sert plus de ses griffes pour se défendre, la civilisation met aux mains des êtres intelligents d'autres armes plus sûres et moins dangereuses. ${ }^{33}$

Mais le raffinement tout « civilisé » du moyen choisi par Nathalie pour tuer sa rivale - elle emploie un poison exotique rare et quasi inconnu, ce qui aura pour conséquence imprévue et fâcheuse de faire accuser Claude à sa place, alors qu'elle espérait le voir revenir vers elle - n'atténue pas l'animalité qui affleure en elle. Souvent comparée à une chatte tout au long du roman, elle se trahit lors d'une scène de jalousie, lorsqu'elle sent Claude lui échapper :

Claude, qui la regardait maintenant en face, fut frappé de l'expression de férocité qui s'était imprimée sur son visage : à la voir ainsi, menaçante, prête à s'élancer, c'était à croire que cette femme, ordinairement si belle, avait, avec quelque bête féroce, une parenté lointaine, dont elle n'était pas encore complètement affranchie. Ils restèrent ainsi durant quelques minutes; puis cette expression de férocité s'adoucit ; le visage reprit son caractère humain, mais sans que les yeux cependant perdissent leur obliquité et leur mobilité. ${ }^{34}$

Claude lui-même, tout innocent qu'il soit, n'est pas exempt de cette pulsion bestiale du meurtre. Lors d'une crise de jalousie, cette fureur a en effet menacé de se retourner contre Nathalie, alors encore sa maîtresse ${ }^{35}$. Mais les apparences peuvent aussi être trompeuses: les frères Vilaine, les deux charbonniers accusés à tort de meurtre et innocentés par la contre-expertise de Claude, avaient pâti d'un préjugé défavorable à cause de leur aspect bestial ${ }^{36}$, de $«$ leur air sauvage $»^{37}$.

Mais si c'est avec Conscience et Justice, ou plus exactement dans le dialogue intertextuel entre ces deux romans, comme nous le verrons, que l'atavisme du meurtre devient un thème central chez Malot, c'est également là qu'il se révèle dans toute sa complexité.

Dans Conscience plusieurs indices tendent à rapprocher Victor Saniel, le médecin meurtrier, du criminel-né, de l'atavique lombrosien. Son aspect physique d'abord, déterminant dans le système du criminaliste italien, est caractéristique :

[...] c'était un grand et solide garçon d'une trentaine d'années, aux cheveux fauves tombant sur le collet de sa redingote, à la barbe longue, frisante, à la figure énergique, mais tourmentée, ravagée, à laquelle des yeux bleu pâle donnaient une expression de dureté que précisait encore une mâchoire osseuse et son allure décidée : en tout un Gaulois, un vrai Gaulois des temps passés, fort, crâne et résolu. ${ }^{38}$

Ce portrait ne correspond pas en tout point à celui du criminel-né selon Lombroso, mais Malot y souligne les traits ataviques, faisant de Saniel comme une survivance d'époques passées $^{39}$. Et ces traits physiques sont encore renforcés par son origine sociale paysanne, sur laquelle le narrateur et le personnage lui-même insistent à plusieurs reprises ${ }^{40}$. En outre, quand, pour ne pas être reconnu d'une femme qui a vu son visage avant qu'il ne commette son meurtre, il se rase les cheveux et la barbe, l'exclamation qui lui échappe 
devant son reflet dans le miroir est éloquente: " - Voilà bien vraiment une gueule d'assassin! $»^{41}$ contentant de souligner « ses fortes mâchoires $»^{49}$ - caractéristiques du criminel-né selon Lombroso. Mais le thème de la frontière dangereusement floue et perméable entre l'animal et l'humain réapparaît aussi lorsque Florentin fait le récit de ses neuf ans de réclusion en Nouvelle Calédonie. Sa résistance et son évasion sont alors présentées comme une lutte contre la déchéance que représente le retour à l'animalités ${ }^{50}$.

Toutefois le profil psychologique de Saniel ne correspond en rien à celui du criminel-né, poussé au meurtre par une pulsion fatale et irrésistible. Le crime perpétré par le docteur est prémédité, réfléchi, calculé jusque dans sa brutalité même, destinée à éloigner le soupçon ${ }^{42}$. Saniel pousse le sang-froid jusqu'à théoriser son acte avant de le commettre, dans des termes qui rappellent évidemment Crime et châtiment de Dostoïevski mais dont les accents darwiniens tendent parfois à nous ramener paradoxalement vers la théorie de l'atavisme criminel ${ }^{43}$.

On le voit, le statut de l'atavisme du meurtre est pour le moins ambigu dans Conscience. Il en va tout autrement dans la suite que Malot donne à ce roman l'année suivante, intitulée Justice. On y retrouve Saniel, neuf ans après ses meurtres, devenu un médecin respecté et influent, mais seul depuis que sa femme l'a quitté en découvrant la vérité. Il se remarie avec une veuve riche qui l'avait consulté pour ses deux enfants, Valérien et Calixte, craignant que ces garçons souffreteux aient hérité de la phtisie qui a emporté leur père. Avant même de devenir leur beau-père, il leur impose des travaux physiques qui les fortifient dans un premier temps, malgré leurs réticences. Mais, après une première alerte - une fièvre scarlatine dont Saniel parvient à guérir Valérien - c'est Calixte, l'aîné, qui tombe malade à son tour et succombe trois semaines plus tard à une fièvre typhoïde, puis Valérien qui meurt enfin de la même maladie moins d'un an après. Saniel est alors accusé d'avoir empoisonné ses deux beaux-fils pour s'approprier leur héritage. Paradoxalement, ce sont ces crimes, qu'il n'a pas commis, qui font ressurgir les véritables meurtres du médecin : il se suicide après avoir appris sa condamnation à la peine capitale ${ }^{44}$.

Dans ce second volet, l'atavisme est clairement invoqué par Saniel lui-même, lorsqu'il tente vainement de justifier son crime auprès de sa femme Philis. Celle-ci est d'autant moins prête à lui pardonner que c'est son propre frère, Florentin, qui a été condamné à la place du médecin à la réclusion à perpétuité, et qu'elle a quitté son mari à la fin de Conscience en apprenant la vérité. Le plaidoyer du médecin est cependant des plus spécieux : il aurait perpétré les deux meurtres sous l'influence de l'atavisme ancestral ${ }^{45}$ - qu'il ne fait remonter qu'à ses origines paysannes, sans aller jusqu'à invoquer d'ascendant plus lointain, préhominien ${ }^{46}-$, mais l'homme civilisé et policé qui demeurait en lui aurait repris le dessus, et éprouvé un repentir sincère ${ }^{47}$. Ce raisonnement ne convainc ni la jeune femme (au moins sur le moment), ni plus tard Florentin qui, évadé du bagne, a juré de faire éclater la vérité et de confondre l'assassin impuni, ce dont Philis tente alors de le dissuader en reprenant les explications de son ex-mari ${ }^{48}$.

Malot revient peu par ailleurs sur les signes physiques d'atavisme chez Saniel, se Si problématiques et peu orthodoxes que soient les emprunts de Malot aux théories lombrosiennes, le passage au premier plan de ce thème et du vocabulaire qui lui est associé est saisissant entre Conscience et Justice: simplement évoqué allusivement dans le premier volet, l'atavisme criminel devient un ressort narratif et explicatif dans le second. 
Comment expliquer cette évolution ? La question des sources est délicate, tant est pauvre la documentation disponible sur la genèse des romans de Malot. Il faut de plus faire la part d'une "version française » de la théorie du criminel-né, à la fois concurrente, souvent proche mais néanmoins différente de celle de Lombroso. Si d'autres sources méritent d'être examinées, on trouve dans la réception critique de Conscience des éléments précieux de ce « dossier ».

En effet, ce n'est peut-être pas tant à Malot lui-même que l'on doit l'importance du thème de l'atavisme dans Justice qu'à son confrère et critique Anatole France (1844-1924). Rendant compte de Conscience dans Le Temps ${ }^{51}$, où le roman avait paru d'abord en feuilleton ${ }^{52}$, l'écrivain prend prétexte de sa publication en volume pour présenter une chronique sur les questions de philosophie et d'anthropologie criminelle qu'il soulève.

Sans doute aiguillonné davantage par l'article de France que par le roman de Malot où, comme nous l'avons vu, le thème de l'atavisme criminel n'est guère que suggéré, le philosophe Charles Renouvier (1815-1903) condamna les deux écrivains dans un long article paru dans La Critique philosophique ${ }^{53}$, revue qu'il avait fondée en 1872 avec son disciple et ami François Pillon (1830-1914).

Chef de file d'un courant philosophique très influent sous la Troisième République, le "néo-criticisme", qui prônait un retour à Kant, Renouvier était l'ennemi déclaré de toutes les doctrines déterministes, qu'elles soient d'inspiration hégélienne ou darwinospenceriennes. Contre le fatalisme qu'il voyait à l'œuvre derrière ces systèmes et ces théories, il défendait l'irréductibilité du libre arbitre. On comprend donc aisément que Lombroso et toute l'anthropologie criminelle contemporaine aient aussi compté parmi ses bêtes noires ${ }^{54}$. S'il dénonce la reprise de la théorie du criminel-né par Malot, puis sa mise en lumière par France, c'est que, selon lui, de telles idées sont plus dangereuses sous une forme littéraire, qui leur offre une publicité accrue ${ }^{55}$.

Anatole France répondit aux remontrances de Renouvier dans un article où l'ironie pointe sous un ton déférent ${ }^{56}$. Il y évoque aussi le rôle du darwinisme dans sa formation intellectuelle, en le présentant comme une erreur de jeunesse. On peut se demander dans quelle mesure Malot avait pour sa part assimilé cette influence ${ }^{57}$.

De son côté, le romancier n'était pas resté en marge du débat que Conscience avait suscité. Il avait cru bon de publier dans Le Temps une lettre en réponse à l'article de France ${ }^{58}$, dans laquelle il annonçait la parution d'une suite intitulée Justice. Ce texte pose la question du rôle joué par les remarques de France dans l'évolution du thème de l'atavisme entre les deux romans. Quoi qu'il en soit, et si Malot a donné davantage d'importance aux théories de l'anthropologie criminelle dans Justice, il ne s'y est pas borné à les décalquer fidèlement. S'il retient de Lombroso (et de certains de ses homologues français, au-delà de leurs divergences) l'idée que le meurtre est la survivance du passé évolutif de l'humanité, il met implicitement en cause l'existence même d'un type criminel à travers une remarque faite par Philis ${ }^{59}$. Anatole France lui non plus ne prenait pas pour argent comptant les théories de l'anthropologie criminelle, en particulier son versant physiognomonique, évidemment attrayant mais cependant souvent critiqué ou subverti par les romanciers. Un des exemples les plus frappants en est d'ailleurs La Bête humaine de Zola, paru un an après Justice. Quand Anatole France donna un compte rendu aussi développé que subtil du célèbre roman de Zola, sous la forme d'un dialogue fictif ${ }^{60}$, nul doute que les débats suscités par les deux romans de Malot étaient encore frais sous sa plume $^{61}$. 
ànt à Malot, lorsqu'il évoqua la genèse de Conscience dans Le roman de mes romans, il se livra à une rationalisation a posteriori où le rôle de la théorie du criminel atavique lui permettait de minimiser l'influence gênante de Crime et châtiment sur son propre roman ${ }^{62}$.

41 Au-delà de la question des sources et des influences, qui mériterait pourtant d'être examinée plus avant, on peut voir à quel point les romans de Malot ont participé à la mise en question des théories de l'anthropologie criminelle, et alimenté les débats qu'elles ont suscités.

\section{NOTES}

1. Soixante selon la biographie établie par Yves Pincet et actualisée par Francis Marcoin (dernière mise à jour le $1^{\text {er }}$ mai 2015): http://www.hector-malot.org/medias/File/Malot\% 20bibliographie\%20mai\%202015.pdf (consulté le 18 septembre 2015, adresse caduque. Nouvelle adresse : http://www.amis-hectormalot.fr/bibliographie/) [Le nouveau site de l'Association des amis d'Hector Malot (http://www.amis-hectormalot.fr/) est en cours de réfection mais n'est pas encore opérationnel]

2. Ainsi que le souligne justement Guillemette Tison, qui signe l'entrée "Malot» dans le Dictionnaire du roman populaire francophone, dirigé par Daniel Compère (préface de Pascal Ory, Paris, Nouveau Monde éditions, 2007, p. 271): «Son œuvre nous offre un véritable témoignage sur la société de la fin du XIx ${ }^{e}$ siècle ».

3. Achille Foville, «La médecine et la folie dans le roman réaliste - Le mari de Charlotte, de M. Hector Malot ", Gazette hebdomadaire de médecine et de chirurgie, $2^{\mathrm{e}}$ série, t. XII, $22^{\mathrm{e}}$ année, ${ }^{\circ}{ }^{\circ} 10,5$ mars 1875, p. 145-149 et n'11, 12 mars 1875, p. 161-166; Ferdinand, Brunetière, «Le roman réaliste en 1875 », La Revue des deux mondes, XLV ${ }^{\mathrm{e}}$ année, vol. VIII, $1^{\mathrm{er}}$ avril 1875, p. 700-713 (repris dans Le roman naturaliste, Paris, Calmann Lévy, 1883, p. 1-28).

4. Je me permets de reprendre le terme que j'avais proposé pour les besoins de ma thèse de doctorat Théories scientifiques et représentations littéraires de l'hérédité en France (1847-1902) : la science dans l'espace public, entre acculturation et appropriation, centre Alexandre Koyré (EHESS), dirigée par Claude Blanckaert et soutenue en 2010. Disponible en impression à la demande auprès de l'ANRT : http://www.diffusiontheses.fr/68762-these-de-kiriow-ivan.html (consulté le 18/11/16).

5. Hector Malot, Le mari de Charlotte, Paris, Michel Lévy frères, 1874. Paru en feuilleton dans Le Bien Public en 1873.

6. Aude Fauvel, « La voix des fous. Hector Malot et les "romans d'asile" ", Romantisme 3/2008 ( ${ }^{\circ}$ 141), p. 51-64. Consultable en ligne: http://www.cairn.info/revue-romantisme-2008-3page-51.htm (consulté le 18/11/16).

7. «Après Un beau-frère Malot écrivit d'abord Le Mari de Charlotte (1873) pour dénoncer à nouveau les dangers de la médecine mentale. Son propos n'y était guère différent : il s'agissait là encore de pointer les limites de la loi de 1838 , le roman racontant comment un homme pouvait se servir des asiles pour se débarrasser d'un mari et profiter de sa femme. / Plus intéressant, en revanche, est le troisième et dernier livre qu'il consacra à l'aliénisme : Mère, paru pour la première fois dans le Figaro en 1889. » (A. Fauvel, op. cit.)

8. Prosper Lucas, Traité philosophique et physiologique de l'hérédité naturelle..., Paris, J.-B. Baillière, 2 tomes, 1847-1850. 
9. «Pas de recours au passé ni d'histoire familiale pour expliquer la folie donc, mais une intrigue totalement au présent. » (Viviane Alix-Leborgne, op. cit., p. 6).

10. H. Malot, Le mari de Charlotte, Michel Lévy frères, 1874, p. 259.

11. Achille Foville, op. cit ; Ferdinand, Brunetière, op. cit

12. « [...] depuis un an j'ai feint la folie. » (Ibid., p. 433).

13. Hector Malot, Mère, Paris, Flammarion, 1896, p. 377-378 [ $1^{\text {re }}$ édition : G. Charpentier, 1890. Paru en feuilleton dans Le Figaro du 29/12/1889 au 18/02/1890])

14. Voir Aude Fauvel, op. cit

15. Pour un résumé de cette théorie, ainsi qu'un aperçu de son influence sur la littérature, voir Lucia Rodler, « L'homme criminel de Cesare Lombroso : entre science et littérature », Criminocorpus [En ligne], Histoire de la criminologie, 4. L'anthropologie criminelle en Europe, mis en ligne le 24 mai 2012, consulté le 25 août 2015. URL : http://criminocorpus.revues.org/1893 (consulté le 20/11/16).

16. Colette Becker, «Zola et Lombroso. À propos de La Bête Humaine », Cahiers naturalistes, $\mathrm{n}^{\circ} 80$, 2006, p. 37-49. Repris in Bertrand Marquer (dir.), Cesare Lombroso e la fine del secolo : la verità dei corpi, Publif@rum, 2004, n², p. 1-14. [En ligne]. URL : http://www.farum.it/publifarumv/n/01/ becker.php_(consulté le 20/11/16).

17. Le roman serait une exception au rejet du pastorisme et de la théorie microbienne des maladies que Jean-Louis Cabanès a observé chez les écrivains réalistes et naturalistes, et qu'il explique en partie par des raisons idéologiques et politiques: ces écrivains soupçonnaient Pasteur de spiritualisme et avaient penché plutôt du côté de Pouchet dans la controverse sur les générations spontanées qui avait opposé les deux savants (J.-L. Cabanès, Le Corps et la Maladie dans les récits réalistes (1856-1893), Klincksieck, Paris, t. II, p. 199-201).

18. H. Malot, Conscience, op. cit., p. 74-75.

19. Ibid., p. 404.

20. Hector Malot, Justice, Paris, G. Charpentier, 1889 (paru en feuilleton dans Le Temps, du 20/12/1888 au 24/2/1889), p. 9-18.

21. «En un mot il y avait en toi un mélange; si tu étais artiste, tu étais aussi par d'autres côtés homme d'affaires. D'où vient ce mélange, je n'en sais rien, de tes ancêtres probablement, et il est chez toi le résultat de l'hérédité ; [...] 》 (H. Malot, Le docteur Claude, 2 vol., Paris, Dentu, 1879, t. I, p. 12)

22. «[...] je me demandais comment deux femmes pouvaient se ressembler autant et en même temps être si différentes l'une de l'autre; je ne dis pas moralement, mais physiquement. / - Affaire d'hérédité, monsieur le docteur [...]. » (Ibid., p. 58).

23. Hector Malot, Un Beau-Frère, Paris, Hetzel, 1869 [parution en feuilleton dans Le Journal des Débats en 1868], p. 273.

24. Ibid., p. 274.

25. H. Malot, Le mari de Charlotte, op. cit., p. 52.

26. Ibid., p. 63.

27. Ibid., p. 82.

28. Ibid., p. 138.

29. M. de la Héraudière évoque (Ibid., p. 173) "l'évêque de Châlons » (Monseigneur Guillaume Meignan (1817-1896), évêque de Châlons-sur-Marne de 1865 à 1884 et auteur en 1869 du livre Le Monde et l'homme primitif selon la Bible) et « l'abbé Lambert » (Edmond Lambert (1826-1886), auteur en 1868 d'un livre intitulé Le déluge mosaïque : l'histoire et la géologie). Rien ne prouve que Malot ait lu ces ouvrages, mais peut-être a-t-il consulté L'homme primitif (1870) de Louis Figuier, où ils sont tous deux cités (voir Claude Blanckaert, «Les bases de la civilisation. Lectures de L'homme primitif de Louis Figuier (1870) », Bulletin de la Société Préhistorique Française, vol. 90, n¹, 1993, p. 31-49).

30. «- Si j'étais fou, dit-il, je vous tuerais et la justice n'aurait rien à dire, puisqu'elle aurait affaire à un fou. » (Le mari de Charlotte, op. cit., p. 433). 
31. «[...] le marquis se rappela que sa mère aimait aussi cette odeur de lait brûlé. / Que signifiait celle ressemblance ? [...] n'y avait-il pas là un cas d'hérédité, que les savants appellent l'atavisme, c'est-à-dire la reproduction chez les petits-enfants des qualités de leur grand-père ou de leur grand-mère ? / Mais alors Denise était donc bien sa fille : il en avait une preuve. / [...] quand il eut trouvé que l'atavisme le faisait le père de Denise, il éprouva un profond soulagement. / Atavisme ! Cela répondait à tout. Ce n'était pas lui qui avait inventé une règle physiologique pour les besoins de l'heure présente, c'était la science qui l'avait formulée depuis longtemps en se basant sur l'expérience : tout le monde sait, sans faire métier de savant, que certaines affections, le rhumatisme, la goutte vont du grand-père au petit-fils. / Quoi de plus concluant?» (Hector Malot, La fille de la comédienne, Paris, Michel Lévy frères, 1875, p. 77-78)

32. F. Brunetière, op. cit, p. 702.

33. Le Docteur Claude, op. cit., t. I, p. 363.

34. Ibid., t. I, p. 375.

35. Ibid., t. I, p. 231.

36. Ibid., t. I, p. 105 et p. 108.

37. Ibid., t. I, p. 99. On ne peut manquer de penser au personnage de Cabuche dans La Bête humaine , brute au grand cœur vivant dans les bois à l'écart de la société, et lui aussi accusé à tort d'un meurtre.

38. Hector Malot, Conscience, op. cit., p. 7.

39. Malot revient plus loin sur « son air gaulois» (p. 253) ainsi que sur « sa nature brutale» (p. 39).

40. «[...] je manque de politesse et suis resté pour beaucoup de choses l'Auvergnat lourd et gauche que la nature m'a fait [...]» (Ibid., p. 67) ; «[...] lui fils de paysan, resté paysan par tant de côtés [...]». (Ibid., p. 379).

41. Ibid., p. 266.

42. «[...] un médecin n'étrangle pas ses clients, il les empoisonne, il les tue scientifiquement, non brutalement. » (Ibid., p. 86). «Cafflé étranglé, ce ne serait pas sur un médecin que les soupçons se porteraient, ce serait sur une brute; quand les médecins veulent tuer quelqu'un, ils opèrent savamment par le poison ou toute autre mort scientifique; les brutes y vont brutalement; le meurtre dit la profession de l'assassin. » (Ibid., p. 94)

43. «-Ce sont les faibles qui se tuent ; les forts luttent jusqu'à leur dernier souffle. » (Ibid., p. 23)

44. Dans sa dernière lettre, adressée à Philis, Saniel, proclamant une dernière fois son innocence dans la mort de ses deux beaux-fils, lui répète sa justification : «Rien ne restait plus en moi de l'homme que vous avez connu : dégagé de l'influence atavique le crime m'était devenu impossible comme à vous. " (Justice, op. cit., p. 422)

45. Hector Malot, Justice, op. cit., p. 72-73.

46. Il justifie cependant son acte en des termes éminemment darwiniens : « Lui ou moi : c'était la bataille de la vie, la lutte pour l'existence engagée de telle sorte que l'épargner c'était me condamner moi-même au suicide.» (Ibid., p. 71). "[...] non un crime par conséquent, mais simplement un acte de violence, celui que commet le fort envers le faible. » (Ibid., p. 72)

47. Ibid., p. 74.

48. Hector Malot, Justice, p. 309-310.

49. Ibid., p. 219.

50. «-Pauvre maman, qui croit que l'éducation sert à quelque chose au bagne; elle est, au contraire, le supplice du condamné, qui souffre d'autant moins qu'il est près de la brute. » (Ibid., p. 100) «Ce que je vous ai dit de la promiscuité de la vie en commun et de son abjection vous fait comprendre ce qu'était la solitude pour moi : le moyen de me ressaisir et de me retrouver, de remonter de la brute à l'homme [...]. » (Ibid., p. 102) 
51. Anatole France, «Les criminels (à propos du nouveau roman de M. Hector Malot, Conscience )", Le Temps, 18 mars 1888, p. 2 ; repris dans La Vie littéraire, $2^{\mathrm{e}}$ série, [Paris, Calmann Lévy, 1890], Euvres complètes, t. VI, Calmann Lévy, Paris, 1926, p. 401-408.

52. C'est d'ailleurs la raison qu'il invoque pour ne pas se livrer à une véritable analyse littéraire du roman : «Je ne me crois pas permis de juger cet ouvrage à la place même où il a paru. » (op. cit, p. 401)

53. Charles Renouvier, "L'Homme criminel de Cesare Lombroso et le criminel systématique de deux romans récents ", La Critique philosophique (Nouvelle série), $4^{\mathrm{e}}$ année, t. II, $\mathrm{n}^{\circ}$ 9, 30 septembre 1888, p. 193-215. Les deux romans en question sont Conscience et Crime et châtiment, qui n'est récent que dans sa traduction française, parue trois ans auparavant ( Le Crime et le châtiment, par Th. [sic] Dostoïevski, traduit du russe par Victor Derély, 1885, 2 vol. in-18 (éd. Plon, Nourrit et C ${ }^{\text {ie }) »,}$ Ibid., note 1, p. 193).

54. Après avoir, de 1874 à 1880, signé une série d'études sur la psychologie de l'homme primitif, où il critiquait les positions évolutionnistes, (Laurent Fedi, «Philosopher et républicaniser : la Critique philosophique de Renouvier et Pillon, 1872-1889 », Romantisme, Paris, Armand Colin, $\mathrm{n}^{\circ} 115$, 2002, p. 65-82 et p. 73, note 30), Renouvier avait déjà publié un article sur le sujet la même année : C. Renouvier, « La Nouvelle école de droit pénal en Italie », Ibid., t. I, n 6, 30 juin 1888, p. 444-463. Toujours en 1888, Pillon avait publié dans la revue les comptes rendus de plusieurs ouvrages traitant d'anthropologie criminelle: on peut donc parler pour cette année d'une véritable campagne menée par La Critique philosophique contre l'anthropologie criminelle, dont Lombroso était la figure emblématique bien que controversée.

55. Renouvier, "L'Homme criminel de Cesare Lombroso et le criminel systématique de deux romans récents ", op. cit, p. 194. Souligné par l'auteur.

56. Anatole France, «La loi morale», Le Temps, 17 mars 1889. L'article est repris, sous le titre « Notice sur Justice », comme postface à l'édition de Justice de 1895 chez Flammarion \& Marpon (dans les CEuvres complètes d'Hector Malot).

57. On sait que Malot possédait dans sa bibliothèque deux livres de Darwin, L'Origine des espèces (Guillaumie \& Cie, 1862) et L'expression des émotions (1874, C. Reinwald), ainsi que de nombreux ouvrages d'histoire naturelle, en particulier plusieurs tomes de La Vie des animaux (Hetzel, 1859-1860, 6 volumes), ouvrage écrit sous le pseudonyme de Johnathan Franklin par l'écrivain Alphonse Esquiros (1812-1876). Je remercie la Société des Amis d'Hector Malot et en particulier Agnès Thomas-Maleville, secrétaire générale, pour les renseignements précieux qu'elle a eu la gentillesse de me communiquer, notamment l'inventaire de la bibliothèque personnelle d'Hector Malot.

58. H. Malot, « Á propos de « Conscience »/Au directeur du Temps », op. cit

59. "Je ne sais s'il y a un type de criminels duquel se rapprochent tous ceux qui commettent un crime...» (H. Malot, Justice, op. cit., p. 309).

60. Anatole France, «Dialogues des Vivants - La Bête humaine », Le Temps, 9 mars 1890 [repris dans La Vie littéraire, $3^{\mathrm{e}}$ série, Paris, Calmann Lévy, 1891], CEuvres complètes, t. VII, Calmann Lévy, Paris, 1926, p. 305-316.

61. Il cite par exemple «Darwin, Maudsley, Lombroso, Henri Joly» (p. 312) comme sources supposées du romancier. Or, concernant Maudsley et Henri Joly, rien n'indique que Zola les ait consultés. Ce sont bien plutôt ses propres sources qu'énumère ici Anatole France, en reprenant celles qu'il avait mobilisées dans ses débats avec Renouvier sur les romans de Malot.

62. H. Malot, Le roman de mes romans, op. cit., p. 243-244. La comparaison entre ce texte et le court article en forme de lettre ouverte au directeur du Temps publié juste après Conscience (« À propos de Conscience/Au directeur du Temps », op.cit) fait apparaître des contradictions entre les deux versions: dans Le roman de mes romans, Malot dit avoir abandonné le projet de Conscience, pourtant déjà mûri selon lui, à cause de la parution de Crime et châtiment, puis l'avoir finalement mené à bien après avoir cédé à l'insistance du directeur du Temps. Il présente par ailleurs 
l'atavisme comme une donnée de départ dans la composition du roman (Le roman de mes romans, p. 248). Malot évoque aussi deux anecdotes relatives à la réception de Conscience, qui montrent surtout à quel point il était resté attaché à l'idée de l'atavisme meurtrier jusqu'en 1896 (Ibid., p. 248-250).

\section{RÉSUMÉS}

Cet article étudie l'investissement littéraire du thème de l'hérédité dans plusieurs romans d'Hector Malot, en particulier sa représentation de la figure du criminel héréditaire ou "atavique ", inspirée de la théorie du criminel-né développée par Cesare Lombroso. Nous verrons aussi comment la réception critique du roman Conscience (1888) et le dialogue entre les disciplines (critique littéraire, philosophie et théories biologiques de l'hérédité) a enrichi et transformé l'approche de cette thématique chez le romancier.

This article studies the litterary investment of the topic of heredity in several of Hector Malot's novels, particularly his representation of the hereditary or « atavistic " criminal, inspired by the theory of the born-criminal developped by Cesare Lombroso. We shall see how the critical reception of the novel Conscience (1888) and the dialogue between disciplines (litterary studies, philosophy and the biological theories of heredity) enriched and transformed the novelist's approach to this theme.

\section{INDEX}

Mots-clés : hérédité, atavisme, Malot (Hector), Lombroso (Cesare), criminel-né

Keywords : heredity, atavism, Malot (Hector), Lombroso (Cesare), born-criminal

\section{AUTEUR}

\section{IVAN KIRIOW}

Docteur de l'EHESS 